\title{
MODELLO DI COMUNICAZIONE NELLA PROSPETTIVA ECCLESIOLOGICA
}

\section{THE MODEL OF THE COMMUNICATION IN THE ECCLESIOLOGICAL PERSPECTIVE}

\begin{abstract}
A b s t r a c t. The article discusses the idea of communication between God and man in the context of the Church, referring to various communication models, and in a special way to the model of understanding and interpretation of Gianni Losito, an Italian sociologist. First, the author tries to explain why this topic is important for contemporary theology and ecclesiology. In the next part of the article there is an attempt to describe and define two key concepts for the subject, namely what we mean by communication and audience (pubblico), which in theological terminology can be understood as a community. As you can see, these concepts are not unambiguous and one concise definition cannot be given due to the multiplicity of forms and ranges of the above-mentioned terms. In the last part there is a short description of different models of interpersonal communication and a wider description of one of them as, according to the author, the most useful and corresponding to the relationship between God and man and between individual human beings. The model of Gianni Losito describes very clearly two phases of the communication process, which is the process of understanding a given message and the process of its interpretation, which means that each person can extract from the given message what is most important or more appealing for him because of his life situation or condition human.
\end{abstract}

Key words: ecclesiology; communication; audience; model of communication; process of communication.

La Trinità creando il mondo e l'uomo e rivelandosi a lui, entrò in un dialogo ininterrotto con lui. Dio, di sua iniziativa, scelse le persone per entrare in una relazione con Lui, ma allo stesso tempo, attraverso queste figure, condusse anche

JACEK Rygielski MIC, dottore di I e II livello, dottorando presso la Facolta di Teologia dell Universita Cattolica di Lublino “Giovanni Paolo II”; e-mail: rygiel119@wp.pl; ORCID: http://orcid. org/0000-0001-7988-3341. 
un dialogo con l'intera comunità. Pertanto, questo dialogo, sebbene rappresenti una relazione personale e unica di ogni uomo con Dio, non può essere separato anche oggi dalla comunità di tutti i credenti, cioè dalla Chiesa, che è lo spazio dell'incontro di Dio con un'altra persona.

La comunicazione tra Dio e l'uomo, che ha usato vari mezzi e persone per questo processo, riceve la sua pienezza con l'Incarnazione di Gesù Cristo, il Figlio di Dio, come dice l'Autore della Lettera agli Ebrei: "Dio, che nel tempo antico avveva parlato ai padri nei profeti, in una successione e varietà di modi, in questa fine dei tempi ha parlato a noi nel Figlio, che egli costituì sovrano padrone di tutte le cose e per mezzo del quale creò l'universo" (Eb 1,1-2). Lo ha sottolineato anche in una delle sue lettere apostoliche alla fine della sua vita, Giovanni Paolo II, scrivendo che "la storia della salvezza racconta e documenta la comunicazione di Dio con l'uomo, comunicazione che utilizza tutte le forme e le modulazioni del comunicare" e poi "la comunicazione tra Dio e l'umanità ha raggiunto dunque la sua perfezione nel Verbo fatto carne. Il Verbo incarnato ci ha lasciato l'esempio di come comunicare con il Padre e con gli uomini, sia vivendo momenti di silenzio e di raccoglimento"2. Il Papa ci ricorda che l'Incarnazione di Cristo è una garanzia non solo di perfetta comunicazione tra l'uomo e Dio, ma anche tra le persone stesse.

Pertanto, la Chiesa, essendo per sua natura una realtà divino-umana e dialogica, nella sua missione utilizza tutti i possibili mezzi di comunicazione sociale (parola, scrittura, immagine, teatro e cinema, radio e televisione, Internet, social media, ecc.) per portare più vicino e corretta comprensione la Rivelazione di Dio. Indubbiamente, un grande impulso a usare questi mezzi, così come il modo del loro uso era uno dei documenti del Concilio Vaticano II Decreto sui mezzi di comunicazione sociale ${ }^{3}$. Questo decreto dice tra l'altro che "la Chiesa cattolica, essendo stata fondata da Cristo Signore per portare la salvezza a tutti gli uomini, ed essendo perciò spinta dall'obbligo di diffondere il messaggio evangelico, ritiene suo dovere servirsi anche degli strumenti di comunicazione sociale per predicare l'annuncio di questa salvezza ed insegnare agli uomini il retto uso di questi strumenti"4.

\footnotetext{
1 Giovanni Paolo II, Lettera apostolica Il rapido sviluppo (Vaticano: Libreria Editrice Vaticana, 2005), n. 3.

2 Ibidem, n. 4.

3 Sobór WaTYKański II, Dekret o środkach społecznego przekazu Inter mirifica (Pallottinum: Poznań, 2002), 87-95.

4 Ibidem, n. 3.
} 
Tuttavia, sembra che la questione più importante non sia tanto la scelta dei mezzi di comunicazione quanto il modello di comunicazione. Questo è fondamentale perché in tale modello possiamo analizzare sia la posizione del mittente che quella del ricevente e seguire l'intero processo di comunicazione e specificare cosa lo determina, facilita o ostacola, così come vedere quali sono i suoi risultati ed effetti. Inoltre, tornando nuovamente al pensiero di Giovanni Paolo II, dobbiamo ricordare che quando parliamo del modello di comunicazione in teologia, dobbiamo tener conto delle sue due dimensioni: verticale (relazione Dio-uomo) e orizzontale (rapporto uomo-uomo), perché "gli interventi di Dio nella storia consistevano sempre nell'incontro di un uomo particolare con Dio e nell'impatto concreto dell'uomo sull'uomo"s.

Pertanto, questo articolo sarà più pertinente alla teologia della comunicazione che alla teoria della comunicazione, anche se queste due realtà si permeano l'una con l'altra. Tuttavia, verrà posta maggiore enfasi sulla relazione tra il mittente e il destinatario, nonché sul modo di trasmissione, ricezione, comprensione e interpretazione del messaggio. Meno invece sulle strategie o l'efficacia della comunicazione tra interlocutori.

Nella moderna teoria della comunicazione, ci sono molti dei suoi modelli che sono stati e sono sistematicamente studiati e sviluppati. Pertanto, nel seguente lavoro, verranno presentate diverse proposte in una prospettiva storica, per mostrare la loro evoluzione e un modello che sembra più appropriato per l'ecclesiologia moderna.

\section{LA COMUNICAZIONE E IL PUBBLICO (COMUNITÀ) - \\ IL TENTATIVO DI CARATTERISTICA}

All'inizio si dovrebbe notare che nelle scienze sociali e nella teoria della comunicazione si usa in modo intercambiabile l'espressione italiana: pubblico e la parola inglese: audience per riferirsi a un gruppo di persone come destinatari di un messaggio specifico. Al contrario, nel linguaggio della teologia, la parola pubblico non viene usata, ma piuttosto comunità che significa: ,unione di persone che hanno un carattere personale e interpersonale, ed è una categoria sociologica, biblica e teologica, oltre che ecumenica. In teologia, comunità significa l'unificazione delle persone basata su relazioni interpersonali autentiche e profonde [...]; il prototipo e la chiamata a costruire una comunità di persone con Dio e le persone

5 Leszek Kuc, Krótki traktat o teologii komunikacji (Leszno: Lumen, 1997), 19. 
tra di loro è la perfetta unità della Santa Trinità e il Suo piano eterno di salvezza dell'uomo nella comunità del Popolo di Dio"6.

Perché sia il concetto di pubblico che di comunità sono vicini al significato, nel mio lavoro, che è nel campo della teologia della comunicazione, userò alternativamente le espressioni del pubblico e dell'audience, prendendo anche in considerazione le connotazioni religiose e teologiche del tema.

Il concetto di comunicazione e il pubblico sono concetti complessi ed è difficile dare una definizione concisa. Entrambi i termini accompagnano le persone nella sua storia, assumono forme diverse e si evolvono costantemente nel corso dei secoli. Questi termini possono essere usati in senso colloquiale nella quotidianità, ma sono anche un oggetto di ricerca in varie discipline scientifiche. In questo articolo saranno presentate in modo sintetico e descrittivo le caratteristiche principali di entrambi i concetti al fine di fornire il loro background significativo.

La comunicazione significa "ogni processo consistente nello scambio di messaggi, attraverso un canale e secondo un codice, tra un sistema (animale, uomo, macchina ecc.) e un altro della stessa natura o di natura diversa"7. Dalla suddetta definizione, possiamo osservare che la comunicazione non è altro che la trasmissione di informazioni ad altri (o ad essere loro destinatari) con il quale consentiamo ad altre persone di essere partecipanti il nostro contenuto mentale o spirituale. Possiamo anche dire che insieme alle informazioni, l'uomo comunica anche se stesso, le sue emozioni, sentimenti, opinioni, ecc.

Nel corso dei secoli, insieme allo sviluppo dei mezzi di trasporto e della tecnologia, stanno cambiando anche le forme di comunicazione. Oggi possiamo contattare una o più persone contemporaneamente senza uscire di casa, anche se queste persone si trovano a migliaia di chilometri da noi. Tuttavia, si deve ammettere che lo sviluppo della tecnologia non presuppone automaticamente lo sviluppo umano personale. E succede che oggi con queste possibilità le persone non si avvicinano necessariamente l'una all'altra e capiscono di più. Pertanto, va ricordato che i vari mezzi tecnici sono solo un aiuto per l'uomo, ma non possono sostituirlo nella costruzione di relazioni interpersonali in cui l'incontro personale, se possibile, delle singole persone è importante.

Con lo sviluppo delle scienze sociali dopo la seconda guerra mondiale, anche i processi di comunicazione sono stati intensamente studiati. All'inizio prevalevano i cosiddetti modelli unidirezionali di comunicazione che erano caratterizzati

6 Antoni NadBrzeżnY, „Wspólnota (w teologii)”, in: Encyklopedia Katolicka, t. 20, editore Edward GigiLewicz (Lublin: Towarzystwo Naukowe Katolickiego Uniwersytetu Lubelskiego, 2014), 1000-1001.

7 "Comunicazione", accesso 24.11.2018, http://www.treccani.it/enciclopedia/comunicazione/. 
dal fatto che l'informazione era passata dal mittente al destinatario, ma senza il cosiddetto feedback. Un esempio qui è il modello di Harold Lasswell (1948) ${ }^{8}$, che descrive l'atto di comunicazione con cinque domande: chi dice - che cosa attraverso quale canale - a chi - con quale effetto? Per questo autore, la comunicazione è principalmente un processo persuasivo che mira a rafforzare, plsamare il nuovo o modificare l'atteggiamento del destinatario.

Altri scienziati (Shannon e Weaver, 1948; Newcomb, 1953; Schramm, 1954; Gerbner, 1956; Lazarsfeld, 1955) ${ }^{9}$ hanno iniziato a prestare attenzione all'enorme complessità dei processi di comunicazione, creando nuovi e più complessi modelli che prendono in considerazione vari fattori aggiuntivi, come ad esempio: rumori nel processo di comunicazione, esperienza di mittente e destinatario, feedback, ecc.

I successivi modelli di comunicazione interpersonale hanno sempre più iniziato ad apprezzare il ruolo e la posizione dei destinatari che diventano partecipanti attivi nel processo di scambio di informazioni. È diventato importante non solo inviare un messaggio, ma anche la sua ricezione, comprensione e risposta (feedback).

Il concetto di pubblico è continuamente oggetto della ricerca empirica svolta soprattutto dagli studiosi della comunicazione di massa e dei media. Il termine pubblico (audience), che "indica semplicemente i lettori, gli spettatori o gli ascoltatori di un canale mediale o di un tipo di contenuto o di spettacolo"10. L'origine del termine è antico, negli spettacoli teatrali e musicali greco-romani, dove tempo e spazio erano precisamente localizzati e definiti, il pubblico (audience) partecipava personalmente agli eventi. Le città antiche avevano sempre un'arena o una piazza dove si riuniva la gente per scopi religiosi, politici o per l'intrattenimento.

In seguito, parallelamente a sviluppi e cambiamenti demografici, economici, sociali, culturali e tecnologici si assiste al continuo cambiamento del concetto di pubblico. Il primo grande passo è avvenuto con l'invenzione della stampa (libro, poi periodici e giornali) con cui si è delineato il pubblico dei media, più vasto e più grande, ma che comprendeva sempre persone in grado di leggere cioè si riferiva soprattutto a una certa classe sociale delle città ${ }^{11}$. Possiamo anche dire

\footnotetext{
${ }^{8}$ Cf. Harold, Lasswell, "The structure and function of communication in society", accesso 25.11.2018, https://pracownik.kul.pl/files/37108/public/Lasswell.pdf.

${ }^{9}$ Franco Lever, Cesare P. Rivoltella, Adriano Zanacchi, "Comunicazione. Dizionario di scienze e tecniche", acesso 25.11.2018, https://www.lacomunicazione.it/voce/comunicazione/.

${ }^{10}$ Denis Mcquail, L'analisi dell'audience (Bologna: Mulino, 2001), 11.

${ }^{11}$ Cf. Carlo Gagliard, "Pubblico", in La comunicazione. Dizionario di scienze e tecniche, editori Franco Lever, Cesare P. Rivoltella, Adriano Zanacchi (Roma: Rai Libri, 2002), 926.
} 
che qui inizia pian piano la comunicazione a distanza e la fruizione individuale. Poi, grazie all'inizio del cinematografo nel 1895, il pubblico condivide la stessa esperienza (non dal vivo, ma sempre identica), supera i limiti dello spazio e del tempo e, inoltre, grazie al cinema e alla radio basta solo guardare e ascoltare, non c'è bisogno di leggere, il che sta a significare la nascita del primo pubblico di mas$\mathrm{sa}^{12}$ poiché allo stesso messaggio o spettacolo possono assistere o partecipare migliaia di persone, sperimentando la stessa esperienza emotiva ed educativa. Altra grande svolta nello sviluppo del termine pubblico è avvenuta con i nuovi media ${ }^{13}$.

Osservando l'evoluzione del termine "pubblico" è da notare che inizialmente tale concetto indicava individui che assistevano a spettacoli o ad altri eventi pubblici come una comunità omogenea, per giungere poi ai pubblici differenziati, frammentati dal punto di vista dei vari tipi di media e ai diversi modi di fruizione.

Bisogna inoltre dire che al termine "pubblico" viene spesso assegnato un significato diverso o confuso con altri termini prelevati dalle scienze della comunicazione e che riguardano altri concetti di comunità. Dunque, per capire meglio il termine "pubblico" è necessario distinguerlo dagli altri concetti e termini di collettività come:

popolazione, insieme delle persone fisiche che risiedono (anche temporaneamente) su un determinato territorio; popolo, insieme delle persone fisiche che hanno la cittadinanza di uno Stato; elettorato, insieme dei cittadini aventi diritto di voto; folla, gruppo di persone numeroso, transitorio, generalmente eterogeneo, formato di individui occasionalmente riuniti fisicamente in un determinato spazio e animati da analoghi interessi e da analghe intenzioni; massa, un insieme non strutturato di persone, la cui eterogeneità interna non dà luogo a forma (almeno attualmente) ${ }^{14}$.

McQuail menziona anche l'esistenza di un gruppo dove "tutti i membri si conoscono e interagiscono tra loro all'interno di determinati confini fisici e sociali, sono consci della loro comune appartenenza e condividono gli stessi valori.

${ }^{12}$ Cf. ibidem, 926.

13 "Le nuove tecnologie mediatiche, o new media, come il videoregistratore, la televisione satellitare e via cavo, Internet, i videogiochi, il cellulare, i CD-Rom, i DVD ecc., contribuiscono alla frammentazione e alla dispersione del pubblico, che impegna il tempo libero a disposizione (time - budget) nell'uso di più media. [...] con i new media si è in presenza di molteplici pubblici, differenziati sulla base dei gusti e degli interessi soggettivi, che non condividono necessariamente gli stessi contenuti". Saveria CAPECCHI, L'audience "attiva". Effetti e usi sociali dei media (Roma: Carocci, 2011), 167.

${ }^{14}$ Vitaliano Rovigatti, Scienza dell'opinione pubblica (Cinisello Balsamo: Edizioni Paoline, 1985), 65-69. 
La struttura delle relazioni del gruppo è chiara e stabile nel tempo"15. A differenza di queste e di altre parole che esprimono collettività, lo stesso autore sottolinea che il pubblico "è il prodotto della condizione moderna, soprattutto se visto come elemento essenziale della democrazia politica. Consiste di un insieme di persone che si impegnano liberamente nella discussione di una questione pubblica, avanzando opinioni, interessi, politiche o proposte di cambiamento" ${ }^{16}$. Invece le caratteristiche principali della nozione "pubblico" sottolineano che l'audience è "disperso e permanente, si forma intorno a una causa e a uno scopo, e il suo primo obiettivo è quello di far progredire un'opinione o un interesse" ${ }^{17}$. Un altro autore, Vitaliano Rovigatti, indica i tratti come "consapevolezza, responsabilità, capacità critica, coerenza"18 dell'audience. Come vediamo è difficile dare una definizione di pubblico perché il termine si sviluppa sempre ed è usato in molti contesti. Bisogna anche dire che il pubblico è sempre molto vasto, ampio e grande e per questo non è facile osservare ed esaminare questo fenomeno se non solo in una sua parte, come per esempio il pubblico della TV o il pubblico di una serie televisiva. Altra difficoltà per definire il pubblico è quella della creazione di nuovi pubblici in quanto la tecnologia si sta sempre più sviluppando, ma anche per i cambiamenti culturali e sociali che sono i fattori importanti del pubblico, dunque, il pubblico o i pubblici sono anche in continuo mutamento, come afferma anche Saveria Capecchi "il concetto di pubblico è complesso ed i suoi significati variano in base ai cambiamenti culturali, economici e tecnologici riguardanti i media, accumulandosi nel tempo" 19 . Per questo il termine pubblico diventa sempre un concetto ambiguo e si possono notare tante differenze di significato.

Da quando gli studiosi si occupano del concetto di pubblico, pongono anche la domanda: l'audience è solo semplice ricevente che accoglie e legge il messaggio così come è stato dato e presentato o l'audience interagisce con testo o contenuto che ha ricevuto?

All'inizio bisogna notare che la concezione dell'audience moderna è diversa rispetto a quella precedente:

non è né prevedibile né pianificabile, è un aggregato casuale, mutevole e provvisorio di individui che fruiscono i media in maniera indiretta: la ricezione dei testi non avvie-

\footnotetext{
${ }^{15}$ MCQUaIL, L'analisi dell'audience, 17-18.

${ }^{16}$ Ibidem, 18.

${ }^{17}$ Mauro Wolf, Teorie delle comunicazioni di massa (Milano: Bompiani, 1991), 28.

${ }^{18}$ Rovigatti, Scienza dell'opinione pubblica, 70.

${ }^{19}$ CAPECCHI, L'audience "attiva”, 164.
} 
ne nello stesso luogo e nello stesso momento in cui essi vengono prodotti e diffusi. L'attività di consumo dell'audience consiste quindi in un insieme di pratiche ed esperienze sociali non istituzionalizzate ${ }^{20}$.

Per molti studiosi (Frank Biocca, Roger Silverstone) oggi la questione dell'audience passiva/attiva non esiste più perché siamo arrivati al punto che non esiste una fruizione totalmente passiva ${ }^{21}$. Il destinatario di un contenuto mediale vedendo le immagini o un testo, ascoltando la voce o la musica della pubblicità, entra nell'interazione e in questo modo il ricevente nella ricezione presenta sempre un certo grado di attività. Sulla base degli studi di Frank Biocca all'audience attiva si possono associare diversi comportamenti nel processo di ricezione dei testi mediali come: selettività (scelta o rifiuto di certi contenuti ricevuti dai media); utilitarismo (scelta razionale dei contenuti sulla base dei propri bisogni che si vuole soddisfare); intenzionalità (elaborazione e strutturazione dei contenuti ricevuti, si riferisce allo stile di consumo); coinvolgimento (individuale o collettivo, emotivo); resistenza o impermeabilità (capacità di opporsi ai contenuti ricevuti) ${ }^{22}$. Un altro autore, Guido Gili, mette in evidenza che "il ricevente è un elemento attivo della comunicazione, non meno dell'emittente" ${ }^{23}$ e la sua attività si esprime attraverso i seguenti concetti: la decodifica, la selettività, l'interpretazione e il coinvolgimento.

La ricerca sull'audience attiva ha rivelato che lo spettatore attivo non deve essere necessariamente adulto infatti anche $\mathrm{i}$ bambini diventano un pubblico attivo dei contenuti mediali a loro rivolti:

se anche bambini sono quindi in grado di interpretare con competenza i contenuti mediali, seppure complessi, di metterli in relazione con la propria vita quotidiana e con le altre attività che svolgono, e di trasformare l'esperienza di fruizione in un'occasione di apprendimento e di acquisizione di risorse, questo viepiù deve valere per gli dpettaotri adulti ${ }^{24}$.

\footnotetext{
${ }^{20}$ CAPECCHI, L'audience “attiva”, 164.

21 "L'idea di un'audience passiva rimane invece storicamente legata all'avvento delle comunicazioni di massa all'inizio del XX secolo, andando a coincidere con la connotazione negativa della «massa» che affollava le grandi città industrializzate". Ibidem, 169.

${ }^{22}$ Cf. Ibidem, 163.

${ }^{23}$ Guido Gili, Fausto Colombo, Comunicazione, cultura, società. L'approccio sociologico alla relazione comunicativa (Brescia: La Scuola, 2012), 31.

${ }^{24}$ Mariagrazia FANCHI, L'audience. Storia e teorie (Roma - Bari: Laterza, 2014), 46.
} 
Il termine pubblico nella sua storia ha subìto tanti cambiamenti e il suo significato e sempre in via di sviluppo. Oggi gli studi sull'audience non mostrano solo la sua attività, ma anche la creatività, la responsabilità e la sua azione performativa. Successivamente, nella mia tesi mi occuperò anche della questione di come il pubblico comprende ed interpreta i contenuti mediali, come reagisce nel confronto con loro.

I due concetti sopra descritti sono anche ampiamente usati in teologia. La Rivelazione di Dio, la Sacra Bibbia e l'Incarnazione del Figlio di Dio sono comunicative. Inoltre, Dio non agisce nel vuoto, ma in uno specifico momento storico e comunica con le singole persone o interi gruppi. Ecco perché vale la pena riflettere e chiedersi qual è il ruolo e la posizione della persona umana o della tutta comunità in questo dialogo che si forma attorno alla chiamata e alla promessa di Dio.

\section{I PROCESSI DI COMPRENSIONE E INTERPRETAZIONE DEL MESSAGGIO (MODELLI DI COMUNICAZIONE)}

La pubblicità, le notizie, il dialogo tra le due o più persone come anche gli altri testi mediali, si inseriscono in tutto il contesto di comunicazione, che significa un processo di trasmettere, informare, rendere partecipi o rendere comune ad altri ciò che è nostro. Il processo di comunicazione del messaggio visivo, uditivo o audiovisivo ha le sue fasi che in generale possiamo presentare come:

- la codifica del messaggio da parte della fonte (emittente);

- la trasmissione del messaggio attraverso un canale;

- la ricezione del messaggio da parte del destinatario;

- la decodifica del messaggio.

A questo punto l'attenzione si sposta sul come si svolge il processo di comprensione e interpretazione del messaggio da parte del pubblico. Il pubblico, decodificando il contenuto mediale, comprende e interpreta il messaggio così come inteso dall'emittente? Il pubblico, inteso come singoli individui, comprende e interpreta il messaggio nello stesso modo? A questo punto della mia tesi mi occuperò di dare una risposta a queste due domande.

L'audience come un ricevente attivo, quando fruisce un testo mediale, entra in un rapporto attivo con esso e cerca il suo significato, per questo come scrive Sonia Livingstone:

quando interpreta un programma, il pubblico non utilizza solo le informazioni contenute nel programma, ma anche le esperienze passate rispetto a quel genere di pro- 
gramma e le esperienze personali rispetto al fenomeno rappresentato [...]. Inoltre, tutte queste differenti fonti d'informazione vengono integrate in un modo complesso e coerente. Così, gli effetti di un programma deipendono dalle modalità con cui il pubblico, interpretandolo, lo trasforma rispetto alla versione originaria ${ }^{25}$.

Secondo questa studiosa il pubblico, decodificando il testo, lo trasforma nel suo significato e senso basandosi sulla sua esperienza personale, sociale e culturale. Gianni Losito, uno studioso che si è a lungo occupato dei processi di comprensione e interpretazione dei diversi testi mediali e del rapporto tra pubblico e testo mediale, dalla sua ricezione fino all'eventuale accoglienza o rifiuto definisce il processo di ricezione come "fruizione" inteso come "l'insieme di tutti i diversi processi che caratterizzano il rapporto con i testi mediali, dall'esposizione, all'allocazione dell'attenzione, dalla percezione alla memorizzazione, dalla comprensione all'interpretazione, fino all'accettazione $\mathrm{o}$ al rifiuto del «messaggio» che si suppone essi propongano" ${ }^{26}$. Vediamo, dunque, come il processo di fruizione sia complesso, ma possiamo anche notare subito in questa definizione due cose importanti: la prima che l'autore suggerisce è che il pubblico non presta la sua attenzione al messaggio per intero, in tutte le sue parti, ma "alloca l'attenzione" in maniera selettiva, sulla base dei suoi interessi. La seconda: l'autore separa nel processo di fruizione il momento della comprensione da quello dell'interpretazione, anche se altri autori trattano questi due termini come sinonimi e usano nelle loro definizioni o una o l'altra in modo intercambiabile.

La prima caratteristica fondamentale nei processi di comprensione e interpretazione è l'attenzione che, come già detto, è selettiva. La selettività dipende dall'interesse del fruitore, dalle sue esperienze e competenze, dalle sue differenze individuali. In alcuni casi il livello di attenzione allocata può essere molto intenso, come quando, per esempio, è rivolta ad un film che qualcuno vede la prima volta o verso un prodotto che viene riconosciuto come ciò che può soddisfare un bisogno. Altre volte l'attenzione è minima, perché i testi trasmessi non sono riconosciuti come significativi Se un fruitore presta attenzione al testo mediale, poi lo percepisce, lo accoglie in modo non solo sensoriale, emotivo, ma mette insieme quello che ha ricevuto e la sua esperienza precedente e personale, come afferma Sonia Livingstone che ho citato prima. Qui bisogna sottolineare che

${ }^{25}$ Sonia Livingstone, Making sense of television. The psychology of audience interpretation (London: Routledge, 1998), 18; CAPECCHI, L'audience "attiva", 209-210.

${ }^{26}$ Gianni Losito, Il potere del pubblico. La fruizione dei mezzi di comunicazione di massa (Roma: Carocci, 2002), 15. 
nella percezione di una stessa immagine "soggetti diversi possono pervenire a risultati percettivi differenti, anche se per tutti valgono le tendenze or ora menzionate"27. L'autore mostra come il pubblico, che si compone di individui, riguardo allo stesso testo mediale può prestare attenzione ai diversi momenti significativi del contenuto e non ad altri perché anche se l'audience appartiene allo stesso contesto sociale e culturale, anche se condivide gli stessi valori e stili di vita, ma è sempre diverso dagli altri a causa della sua personalità e dei suoi atteggiamenti individuali.

Tornando al tema della comprensione e interpretazione del messaggio mediale occorre dire che è la tendenza alla rilevanza di comprensione che significa "la corrispondenza tra codifica e decodifica veniva considerata come condizione necessaria, ancorché non sufficiente, per il successo del processo d'influenza, processo esplicitamente e intenzionalmente finalizzato a indurre un mutamento d'opinione e/o di atteggiamento e/o di comportamento nel destinatario"28. Il concetto di comprensione così formulato si riferisce all'informazione trasmessa in modo lineare (dall'emittente che codifica il messaggio e lo trasmette attraverso un canale al ricevente che lo decodifica e accoglie) ed è importante per la persuasione. Una delle prime teorie della persuasione che tiene conto della ricezione del messaggio è stata elaborata da Carl Hovland e dai suoi collaboratori negli anni cinquanta ${ }^{29}$. Per loro è importante la comprensione del messaggio e per questo essi volevano individuare le caratteristiche che la favorivano. Graficamente il concetto si può mostrare $\operatorname{cosi}^{30}$ :

Figura 1. Modello di comunicazione di Carl Hovland (Fabris, 1968)

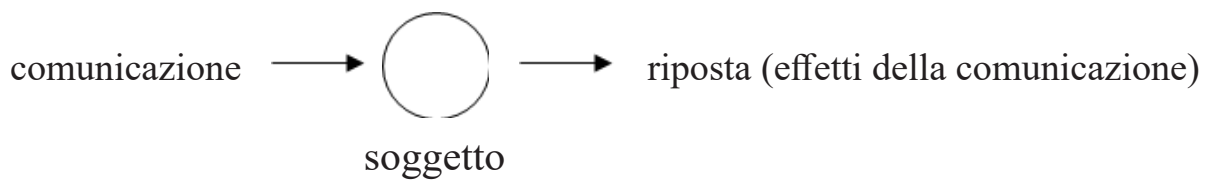

In questo schema vediamo che la comunicazione passa attraverso "la personalità del soggetto stimolandone le relazioni che possono andare da un cambiamento di opinione e di atteggiamento ad una modifica nel comportamento"31. Secondo questo schema non c'è posto per l'interpretazione, ma solo per l'accettazione,

\footnotetext{
${ }^{27}$ Ibidem, 61.

${ }^{28}$ Ibidem, 79.

${ }^{29} \mathrm{Cf}$. Ibidem, 79 .

${ }^{30}$ Giampaolo Fabris, La comunicazione pubblicitaria (Etas/Kompass: Milano, 1968), 17.

${ }^{31}$ Ibidem, 16.
} 
la memorizzazione e la risposta. Così possiamo constatare che qui la comprensione ha un ruolo più importante e l'audience è intesa come più passiva che attiva, cioè il destinatario non elabora i contenuti in modo autonomo, ma accoglie il messaggio così com'è stato dato dall'emittente.

\section{MODELLO DI GIANNI LOSITO}

Lo studioso italiano, Gianni Losito, propone un modello del processo di fruizione dei testi mediali basandosi sulla distinzione tra comprensione e interpretazione, dove "l'una considerata come un processo analitico di «lettura» sintattica e semantica e l'altra come un processo sintetico di attribuzione di un senso unitario al testo considerato nella sua globalità" 32 . All'inizio questa distinzione è stata spesso trascurata, ma si possono anche individuare alcuni contributi di altri studiosi alla ricerca di tali processi come per esempio Luciano Arcuri e Luigi Castelli, anche se nei loro studi non appare esplicitamente il termine interpretazione, tuttavia essi parlano dei diversi livelli della comprensione:

è stato dimostrato che, anche durante queste prime fasi di comprensione, le precedenti conoscenze e gli elementi contestuali presenti intervengono per definire il significato appropriato da attribuire a ciascun termine [...]. Un passo oltre, la comprensione di un testo viene raggiunta quando il lettore - ascoltatore è in grado di stabilire dei nessi causali, di arguire i motivi e le conseguenze di ciò che viene descritto, di assumere il punto di vista del protagonista e di chi invia il messaggio per poter estrarre il nucleo concettuale, o la morale, del messaggio stesso $\mathrm{o}^{33}$.

Un altro contributo al tema viene da Marino Livolsi che fa una chiara distinzione fra i processi di comprensione e interpretazione:

l'incontro testo - lettore avvenga prevalentemente nelle due opposte situazioni del "consumo" e della "ricerca - costruzione" di significato o senso. Nel primo caso esso è fruizione passiva o scarsamente motivata e prevalentemente "guidata" dal testo e dai suoi "suggerimenti" espliciti e non è facilmente oggetto di letture approfondite o alternative. In questo caso il lavoro di decodifica resta, di norma, al livello deno-

\footnotetext{
${ }^{32}$ Losito, Il potere del pubblico, 138.

${ }^{33}$ Luciano ArCuri, Luigi Castelli, La trasmissione dei pensieri. Un aproccio psicologico alle comunicazioni di massa (Padova: Decibel editrice, 1996), 143-144.
} 
tativo (ciò che afferma esplicitamente il testo) e della comprensione, cioè il lettore si limita a comprendere ciò che il testo vuol significare immediatamente e semplicemente. Nel secondo caso, il lettore fa una fruizione attiva, usa il testo come stimolo per un lavoro di interpretazione al livello connotativo (ciò che suggerisce il testo come significato possibile o più generale), sulla base di un continuo processo associativo (di inferenza) che lega alcuni elementi di quel testo a schemi cognitivo - emotivi già in suo possesso ${ }^{34}$.

Il pensiero di questo autore per quanto riguarda i processi di comprensione e interpretazione è simile a quello di Gianni Losito in riferimento ai due termini, come due operazioni legate ma diverse, ma ci sono anche differenze fra questi autori. Livolsi per comprensione intende un processo passivo nel senso che in questo livello il ricevente decodifica solo il messaggio. Invece Losito questa tappa la intende come un'operazione attiva "da coinvolgere entrambi i piani della denotazione e della connotazione. Questi due piani sono continuamente compresenti e intrinsecamente congiunti in ogni testo, anche nel più semplice, e [...] difficile assumere che il fruitore possa operare nella decodifica riferendosi all'uno e ignorando l'altro"35. Losito sostiene che anche se questi due processi sono distinti, entrambi sono attivi e in un certo modo sono complementari ma non sovrapposti.

Per quanto riguarda l'interpretazione i due autori sono più vicini e affermano che quello che distingue la comprensione dall'interpretazione è un valore aggiunto, "il plus che si realizza sia per il processo che ha permesso di trarre un significato dal testo non immediatamente evidente, sia per l'aver effettuato una lettura personale e soggettiva, spesso sottolineata in senso emotivo" "36. Gianni Losito è d'accordo con un plus nel processo di interpretazione che secondo lui è presente già a livello della comprensione.

Lo stesso autore propone un modello per la spiegazione ${ }^{37}$ del processo di comprensione e interpretazione nel seguente modo:

\footnotetext{
${ }^{34}$ Marino Livolsi, Manuale di sociologia della comunicazione (Roma-Bari: Laterza, 2000), 253-254.

${ }^{35}$ Losito, Il potere del pubblico, 139.

${ }^{36}$ Livolsi, Manuale di sociologia della comunicazione, 254.

${ }^{37}$ Cf. Losito, Il potere del pubblico, 141-144.
} 
Figura 2. Modello di comprensione e interpretazione di Losito (2002)

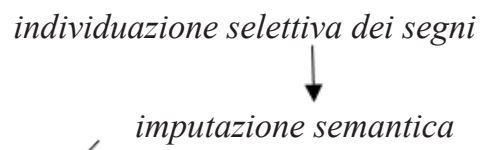

riconoscimento dei significati attribuzione autonoma dei significati attribuzione di senso ricomposizione scoperta

testo $\longrightarrow$ comprensione $\longrightarrow$ "meta-testo" $\longrightarrow$ interpretazione $\longrightarrow$ "messaggio" $\rightarrow$ effetti

Secondo questo schema tutto il processo di comprensione e interpretazione inizia con "l'individuazione selettiva dei segni" ciò significa che in un testo mediale il fruitore alcuni segni li riconosce come significativi e altri no e poi attribuisce a questi segni maggiore o minore rilevanza. In seguito, "l'imputazione semantica" ha due diverse modalità: "il riconoscimento dei significati" da parte del fruitore nel testo mediale a segni prima selezionati e "l'attribuzione autonoma dei significati" che crea il fruitore. Delle due modalità della comprensione può prevalere "il riconoscimento nel caso di un'elevata omogeneità dei livelli di competenza comunicativa e di consonanza culturale e può prevalere invece l'attribuzione autonoma dei significati nel caso di una consistente diversità tra questi stessi livelli" ${ }^{38}$. Altra tappa della comprensione del testo mediale è "la ricomposizione" che significa mettere in relazione i significati prima individuati per creare un nuovo testo che si chiama "il meta - testo" che è figlio ma non clone del testo originario che riduce la sua complessità iniziale ${ }^{39}$.

Qui termina il processo di comprensione e inizia il processo di interpretazione con "l'attribuzione di senso" al "meta - testo". Il materiale per l'interpretazione come vediamo fornisce "l'imputazione semantica". Grazie a questi significati il fruitore può scoprire un messaggio nel testo, un senso profondo, una morale. "La scoperta" del messaggio significa cercare e trovare quello che il testo vuole dire e non necessariamente questo messaggio deve essere inscritto nel testo, può essere implicito. Come ultimo elemento nel modello del processo di comprensione e interpretazione di Losito ci sono i possibili "effetti". Ma come vediamo sul grafico gli effetti non sono frutto del testo mediale in sé ma possono essere semmai il frutto di "meta - testo" come "immateriale esito del processo

${ }^{38}$ Ibidem, 142.

${ }^{39}$ Cf. Ibidem, 142. 
di comprensione, il senso complessivo attribuito ad esso nel processo di interpretazione e il «messaggio» eventualmente scoperto in esso dal fruitore" ${ }^{40}$. Per l'autore il fruitore è un destinatario attivo che, confrontandosi con il testo mediale, costruisce il suo significato.

Dunque, l'interpretazione non significa solo scoprire un senso nascosto del messaggio, ma attribuire anche un significato nuovo sulla base del contesto in cui il testo mediale è stato prodotto, e sulla base del contesto socio - culturale del ricevente in cui il testo viene letto o decodificato. Guido Gili, sulla base della riflessione ermeneutica e antropologica, sostiene che "l'interpretazione avvenga sempre entro «cornici» e «schemi» interpretativi" e che "la nostra esperienza quotidiana è guidata da tali schemi interpretativi che ci consentono di attribuire un significato alle situazioni e alle relazioni sociali in cui siamo coinvolti o che osserviamo"41. Invece Sonia Livingstone sottolinea che "l'interpretazione è il processo di negoziazione tra le possibilità esistenti nel mondo esterno e il repertorio individuale di conoscenze e strategie di elaborazioni individuali" ${ }^{42}$.

Il modello di Gianni Losito in cui sono chiaramente distinti due parti integranti di un processo di comunicazione, sottolinea la relazione unica tra il mittente e il destinatario. Da un lato, non li mette su un piano di parità in questo processo, ma dall'altro, dà molta autonomia e libertà di entrambe le parti nel dialogo. Guardando la Bibbia, che è, in un certo senso, la storia di un dialogo vivo e continuo tra Dio e l'uomo, vediamo che con questo modello possiamo descrivere come appare questa relazione. Dio non vuole che l'uomo sia solo un passivo destinatario e appaltatore della Sua parola, ma vuole impegnare sia la mente che la volontà e il cuore, richiede non solo il ricevimento ma anche l'accettazione della parola, l'adesione ad essa e la risposta che non pone fine al processo di comunicazione, ma lo sostiene e lo rafforza.

\section{RIASSUNTO}

Così come è difficile dare una definizione sufficiente di comunicazione, è anche difficile scegliere uno dei suoi modelli sufficienti e soddisfacenti. Un aspetto importante dei modelli contemporanei è quello di apprezzare non solo il destinatario che accetta attivamente il contenuto trasmesso, cerca di capirlo e interpretarlo,

\footnotetext{
${ }^{40}$ Ibidem, 144.

${ }^{41}$ Gili, Colombo, Comunicazione, cultura, società, 43.

${ }^{42}$ Livingstone, Lo spettatore intraprendente, 31.
} 
ma anche un momento importante è quello di dare una risposta, che si traduce nello stabilire una relazione tra il mittente e il destinatario.

Penso che il modello di Gianni Losito, presentato in dettaglio in questo articolo, possa essere ampiamente usato nella teologia contemporanea e specialmente in ecclesiologia. Nella teoria data da questo autore, vediamo che il destinatario del messaggio, anche se in un gruppo, è chiamato ad accettare, comprendere e interpretare il testo personalmente. Inoltre, anche quando le persone vedono o sentono lo stesso testo, lo capiscono allo stesso modo, ma ciò che tutti prestano attenzione e interpretazione può essere diversa perché è influenzato da molti fattori personali come: emozioni, stile di vita, età, esperienza di vita, situazione attuale in cui qualcuno è ecc.

Nella relazione Dio - uomo non siamo parti uguali di questo dialogo, perché Dio ci supera infinitamente. Tuttavia, vuole che le persone partecipino attivamente a questo dialogo e parlando ad un uomo, richiede che accetti la sua parola, la capisca, la interpreti e dia una risposta libera. Allo stesso modo, nella relazione Chiesa - uomo, dove la Chiesa annuncia la Parola di Dio, chiede la conversione, che richiede una partecipazione attiva e le risposte dei singoli credenti. La teoria della comunicazione contemporanea e la teologia della comunicazione ci mostrano che la comunicazione non è solo una semplice trasmissione di informazioni dal mittente al destinatario. Piuttosto, dovremmo vedere la comunicazione come una relazione tra persone diverse, che richiede rispetto reciproco, ascolto, accettazione e libertà. E perché è necessario cercare questi modelli di comunicazione, anche in ecclesiologia, che garantiscano la conservazione di queste caratteristiche.

\section{BIBLIOGRAFIA}

Giovanni Paolo II. Lettera apostolica Il rapido sviluppo. Vaticano: Libreria Editrice Vaticana, 2005.

Sobór WaTYKaŃSKi II. Dekret o środkach społecznego przekazu Inter mirifica. Pallottinum: Poznań, 2002.

Arcuri, Luciano, CAStelli, Luigi. La trasmissione dei pensieri. Un aproccio psicologico alle comunicazioni di massa. Padova: Decibel editrice, 1996.

CAPECCHI, Saveria. L'audience "attiva". Effetti e usi sociali dei media. Roma: Carocci, 2011.

FABRIS, Giampaolo. La comunicazione pubblicitaria. Milano: Etas/Kompass, 1968.

FANCHI, Mariagrazia. L'audience. Storia e teorie. Roma-Bari: Laterza, 2014. 
Gagliardi, Carlo. "Pubblico". In La comunicazione, Dizionario di scienze e tecniche, editori Franco Lever, Cesare P. Rivoltella, Adriano Zanacchi, 926-930. Roma: Rai Libri, 2002.

Gili, Guido, Colombo, Fausto. Comunicazione, cultura, società. L'approccio sociologico alla relazione comunicativa. Brescia: La Scuola, 2012.

Kuc, Leszek. Krótki traktat o teologii komunikacji. Leszno: Lumen, 1997.

Livingstone, Sonia. Making sense of television. The psychology of audience interpretation. London: Routledge, 1998.

Livolsi, Marino. Manuale di sociologia della comunicazione. Roma-Bari: Laterza, 2000.

Losito, Gianni. Il potere del pubblico. La fruizione dei mezzi di comunicazione di massa. Roma: Carocci, 2002.

McQuaIL, Denis. L'analisi dell'audience. Bologna: Mulino, 2001.

NADBRZEŻNy, Antoni. „Wspólnota (w teologii)”. In: Encyklopedia Katolicka, t. 20, editore Edward GigILEwICZ, 1000-1001. Lublin: Towarzystwo Naukowe Katolickiego Uniwersytetu Lubelskiego, 2014.

Rovigatti, Vitaliano. Scienza dell'opinione pubblica. Cinisello Balsamo: Edizioni Paoline, 1985.

Wolf, Mauro. Teorie delle comunicazioni di massa. Milano: Bompiani, 1991.

\section{WEBGRAFIA}

“Comunicazione”, accesso 24.11.2018, http://www.treccani.it/enciclopedia/comunicazione/.

LASSWELL, Harold. "The structure and function of communication in society", accesso 25.11.2018, https://pracownik.kul.pl/files/37108/public/Lasswell.pdf.

Lever, Franco, Rivoltella, Cesare P., Zanacchi, Adriano. "Comunicazione. Dizionario di scienze e tecniche”, acesso 25.11.2018, https://www.lacomunicazione.it/voce/ comunicazione/.

MODEL KOMUNIKACJI W PERSPEKTYWIE EKLZEJOLOGII

S T R ES Z C Z E N I E

Artykuł omawia ideę komunikacji pomiędzy Bogiem a człowiekiem w kontekście Kościoła, odwołując się do różnych modeli komunikacji, a w sposób szczególny do modelu rozumienia i interpretacji Gianni Losito, socjologa włoskiego. Najpierw autor stara się wyjaśnić, dlaczego temat ten jest ważny dla współczesnej teologii i eklezjologii. W kolejnej części artykułu następuje próba opisania i definicji dwóch kluczowych pojęć dla tematu, a mianowicie, co rozumiemy przez słowo 
„komunikacja” oraz „publiczność”, co w terminologii teologicznej możemy rozumieć jako wspólnota. Jak można zauważyć, pojęcia te nie są jednoznaczne i nie da się podać jednej, zwięzłej definicji ze względu na wielość form i zakresów wyżej wymienionych terminów. W ostatniej części następuje krótka charakterystyka różnych modeli komunikacji międzyludzkiej oraz szersze opisanie jednego z nich jako, według autora, najbardziej przydatnego i odpowiadającego relacjom pomiędzy Bogiem a człowiekiem oraz pomiędzy poszczególnymi osobami ludzkimi. Model Gianni Losito opisuje bardzo wyraźnie dwie fazy procesu komunikacji, jakim jest proces rozumienia danego komunikatu i proces jego interpretacji, co powoduje, że każda osoba może wydobyć z danego komunikatu to, co dla niej najważniejsze lub bardziej przemawiające ze względu na jej sytuację życiową czy kondycję ludzką.

Słowa kluczowe: eklezjologia; komunikacja; publiczność; model komunikacji; proces komunikacji. 